\title{
Characteristics Analysis of Joint Acoustic Echo and Noise Suppression in Periodic Drillstring Waveguide
}

\author{
Li Cheng, ${ }^{1,2}$ Chang Jinfeng, ${ }^{1,2}$ Liu Zhao, ${ }^{1,2}$ Fan Shangchun, ${ }^{1,2}$ and Ding Tianhuai ${ }^{3}$ \\ ${ }^{1}$ State Key Laboratory of Virtual Reality Technology and System, Beihang University, Beijing 100191, China \\ ${ }^{2}$ Science and Technology on Inertial Laboratory, Beihang University, Beijing 100191, China \\ ${ }^{3}$ Department of Precision Instruments and Mechanology, Tsinghua University, Beijing 100086, China
}

Correspondence should be addressed to Li Cheng; lc.bj@163.com

Received 2 January 2014; Revised 11 April 2014; Accepted 28 April 2014; Published 11 May 2014

Academic Editor: Kenneth J. Loh

Copyright (C) $2014 \mathrm{Li}$ Cheng et al. This is an open access article distributed under the Creative Commons Attribution License, which permits unrestricted use, distribution, and reproduction in any medium, provided the original work is properly cited.

\begin{abstract}
A new method of wireless data telemetry used by oil industry uses compressional acoustic waves to transmit downhole information from the bottom hole to the surface. Unfortunately, acoustic echoes and drilling vibration noises in periodic drillstring are a major issue in transmission performance. A combined acoustic echo and noise suppression method based on wave motion characteristic in drillstring is adopted to enhance an upward-going transmitted acoustic signal. The presented scheme consists of a primary acoustic echo canceller using an array of two accelerometers for dealing with the downward-going noises and a secondary acoustic insulation structure for restraining the upward-going vibration noises. Furthermore, the secondary acoustic insulation structure exhibits a banded and dispersive spectral structure because of periodic groove configuration. By using a finite-differential algorithm for the one-dimensional propagation of longitudinal waves, acoustic receiving characteristics of transmitted signals are simulated with additive Gaussian noise in a periodic pipe structure of limited length to investigate the effects on transmission performance optimization. The results reveal that the proposed scheme can achieve a much lower error bit ratio over a specified acoustic isolation frequency range with a $30-40 \mathrm{~dB}$ reduction in the average noise level compared to traditional single-receiver scheme.
\end{abstract}

\section{Introduction}

In the oil industry, measurement-while-drilling (MWD) surveying is of great importance for the successful completion of the drilling process [1]. Such information, if transmitted to the surface, can be used to optimize the drilling by adjusting the direction of drilling and to understand downhole formation conditions in real time [2]. These useful data related to subsurface earth formation can be derived in a number of ways [3]. The common approaches are referred to as either MWD or logging while drilling (LWD), because downhole information may be acquired quicker and at lower cost by use of such techniques [4]. Currently, the telemetry methods available for MWD and LWD tools primarily include mud pulse telemetry, extremely low-frequency electromagnetic (EM) telemetry, high-speed wired pipe telemetry, and drillstring acoustic telemetry. The mud pulse telemetry is the most commercially successful method. However, the data transmission rate is limited to a few bits per second due to attenuations and spreading of pulses. When highly compressible underbalanced drilling fluid is used, such an approach may become ineffective [5]. EM telemetry has been considered for MWD services, but EM signals also encounter high attenuation in regions of low formation resistivity, in cased holes, and where borehole fluid is highly conductive. Namely, it is considered to have limited range, depends on characteristics, especially resistivity, of the formations surrounding the borehole, and also has limited data rates [6]. High-speed wired pipe telemetry can be implemented by using a unique system of wired drill pipes and associated drilling tools connecting the MWD string to the surface. Because special drill pipes and special tool joint connectors are required, the cost of the drilling operation will be substantially increased. The data transmission along the drillstring via acoustic stress waves offers another communication possibility. As early as 
1948, acoustic telemetry was identified as a potential method for high-speed communication. In fact, the drillstring does not have a uniform cross-sectional area, and individual pipes are joined together at 10 meter intervals with heavy threaded couplings [7]. Therefore, the transmission characteristics exhibit a banded and dispersive structure based on the theoretical analysis of an idealized drillstring presented by Barnes and Kirkwood in 1972 [8]. Compared with the other transmission methods, drillstring acoustic telemetry has the advantages to potentially provide high data rate and to be relatively unaffected by the formation properties. Furthermore, a unique multinode acoustic telemetry network capable of transmitting data at over 30 bits per second has been developed and successfully deployed in drilling application [9]. In this case, drillstring waves can be measured for the transmission of information from the drill bit to the surface. Unfortunately, a serious problem related to the transmission of acoustic data while drilling is that ongoing drilling operations tend to generate wide bandwidth noise which contaminates the acoustic data transmission channel through which acoustic data are being transferred. One source of such noises is the drill bit typically located at the end of the drillstring, which will generate stronger upwardgoing drilling noises interfering with the required upward travelling acoustic data. Another is the top drive or other mechanical equipment located at the surface and coupled to the drillstring, which will generate downward-going surface noises interfering with the uplink acoustic data. Due to the acoustic nature, the transmitted acoustic data are disturbed by short- and long-period reverberations produced by the multiple reflections occurring in the drillstring. Regardless of the source thereof, such noises strongly deteriorate the signal-to-noise ratio (SNR), thereby making the data difficult to identify and analyze for this type of acoustic telemetry. Therefore, acoustic echo and noise suppression is a more critical problem at multiple acoustic impedance mismatch positions in drillstring where acoustic waves travel freely up and down the drillstring and confuse the transmission data.

With regard to the aforementioned problem, the theoretical models and schemes concerning acoustic echo or downhole noise suppression have been independently studied to some extent. As far as acoustic echo cancellation is concerned, Rector and Marion used a data-dependent deconvolution operator to perform the inverse filtering of the multiple acoustic reflections [10], which may cause signal distortions due to coherent noise in surface measurements. Drumheller and Scott demonstrated a hardware design method of echo suppression using a novel digital time delay circuit and a pair of spaced sensors such as strain gages or accelerometers [11]. The exact time-delay control of travelling waves between the two sensors and adaptive filters is required in this method. Poletto presented a dual-sensor-based reverberation suppression analysis method by measuring acceleration and strain with opposite reflection coefficients [12]. Addition of the dual waves makes it possible to remove part of the drillstring reflections, but it is prone to reduce the one-way reflection noise from one end of drillstring when the receivers are at intermediate positions. Sinanović et al. described a theoretical channel model and analyzed two-receiver scheme that exploited the fact that the dominant surface noise source and the signal, respectively, propagate in up and down directions [13]. Different delays between the two signals at two receivers are exploited to suppress the downlink surface noise by applying appropriate filters in this model. However, it only takes into account first-order wave reflections at the pipe ends for simplicity and will become complicated when multiple reflections are considered. Besides, downhole noise from drill bit tends to generate wide bandwidth noise which degrades the acoustic transmission performance of data. Meehan demonstrated that a baffle filter, comprising a periodic structure of typically $20 \mathrm{~m}$ interposed above or below the acoustic sub, can reduce the effects of drill bit noise on modulated torsional wave [14]. However, the scheme does not address the more serious problem of downlink wave being reflected in a relatively unattenuated way back to the transmitter where it may deteriorate the uplink acoustic telemetry wave. According to the wave reflection phenomena caused by acoustic impedance mismatches, Drumheller et al. disclosed an acoustic isolator containing multiple tubular members in series connection to mitigate the potentially destructive reflections from interfering with the up wave [15]. This disclosed scheme requires the application of tuning bars whose lengths differ from an odd multiple of a quarter wavelength of a representative acoustic wave, and downhole noise isolator in a predetermined range of acoustic frequencies is not considered. Then Shah et al. introduced multiple dampening members including one or a group of two or more nested cylindrical sleeves in radial direction to configure a low-frequency downhole acoustic attenuator to weaken acoustic signals at frequencies below $5 \mathrm{kHz}$ [16], but a complicated acoustic insulation structure (AIS) is required, and especially its radial dimension is yet confined by drilled borehole size. Several other patents have also been presented on acoustic isolators, but most of them are specially used for seismic signal transmitted through the formations instead of acoustic signal induced in drillstring. Furthermore, their acoustic models, physical structures, and sound insulation performance are not provided in detail. In this paper, considering the effects of downhole/surface noises and periodic drillstring channel, an improved joint echo cancellation and noise suppression method is developed to optimize the communication signal received at the receiver based on multiple extensional wave transmission characteristics in uplink and downlink drillstring channel.

\section{Method Architecture}

The application of acoustic telemetry in real-time drilling situations is much more challenging. This is primarily due to the increased noise from surface equipment and drilling operations and the problem of unwanted acoustic wave reflections associated with beam-like drillstring structure and downhole components, such as the bottom-hole assembly (BHA), typically attached to the end of the drillstring [17]. The drill bit crushes the formation and creates compressional acoustic waves propagating in the drillstring. Normal drilling operations also produce in-band acoustic noise at multiple 


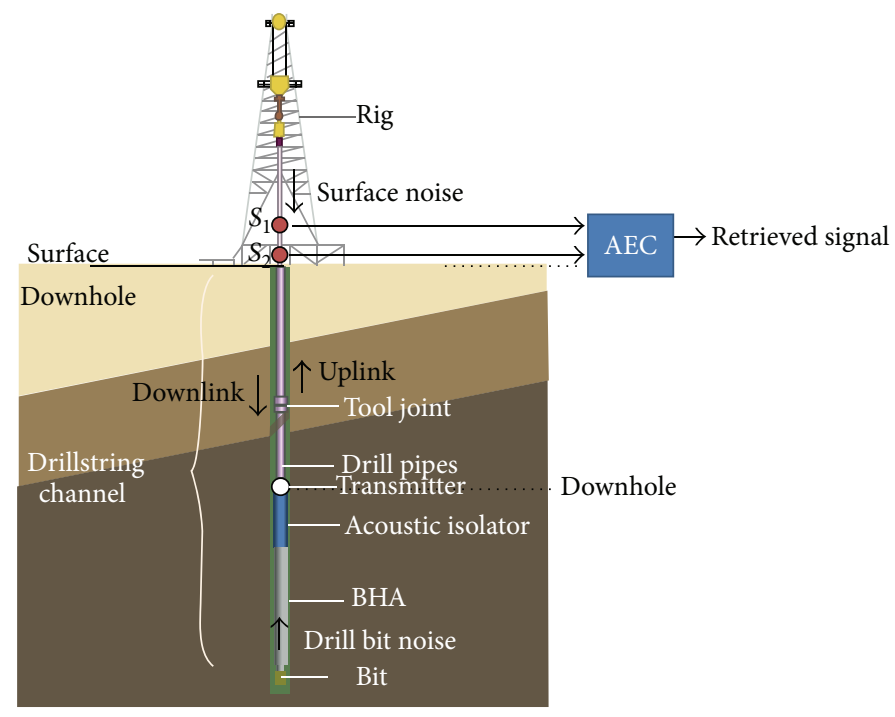

FIGURE 1: Schematic view of the proposed model.

sources at intensities comparable to the acoustic transducer output. The surface noise from surface drilling operations further degrades the signal sent by the transmitter. We use the idealized model as depicted in Figure 1 to analyze the performance of acoustic telemetry.

According to the schematic model, two acoustic receivers may be positioned at or near the top end of a drillstring at the surface in the well. Both the bit noise $n_{d}(t)$ and surface noise $n_{s}(t)$ seriously confuse the transmission of original excitation signal $x(t)$ during the drilling. On one hand, the noise $n_{d}(t)$ travelling upward along the BHA is mixed with the output signal of the transmitter. On the other hand, the noise $n_{s}(t)$ travelling downward along the drillstring is received by the receiver. Considering the fact that compressional acoustic waves travel in two directions in the drillstring, the mixed reverberation signal formed by acoustic signals $x(t), n_{d}(t)$, and $n_{s}(t)$ reaches the receiver in the multiple-path drill pipes. In accordance with the uplink and downlink transmission paths of acoustic waves, an acoustic echo canceller (AEC) and an AIS are, respectively, introduced at the sides of receiver and the transmitter as shown in Figure 1 to obtain higher SNR and lower bit error ratio (BER).

2.1. Use of Dual Waves for Cancelling Downlink Echoes. Acoustic telemetry signals are disturbed by multiple reverberations produced by the reflections occurring at acoustic impedance mismatch positions. Dual measurements record reflected waves travelling in the same direction and make it possible to remove reflections of drillstring waves and unwanted noises. For simplicity, the effects of drill bit noise $n_{d}(t)$ and corresponding acoustic isolator are ignored. Referring to Figure 2 for simplicity, the two acoustic receivers $S_{1}$ and $S_{2}$ spaced one-quarter wavelength of the carrier frequency apart are placed on the first pipe at the top of the drillstring to record two signals, $n_{s}(t)$ and $x(t)$, and their reflections. The noise $n_{s}(t)$ consists of the direct surface noise as well as the downlink echoes of mixed signals from

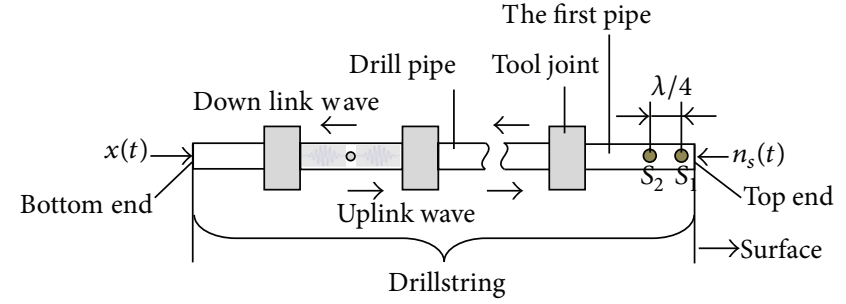

FIGURE 2: Schematic diagram of the AEC using two receivers.

the direct surface noise and the original excitation signal $x(t)$. As a result of the standing wave theory, the receiver $S_{1}$ is located approximately near the top end of drillstring, and the lower receiver $S_{2}$ is $\lambda / 4$ away from the receiver $S_{1}$. Considering the effects of multiple reflections in drillstring channel, the uplink and downlink channel responses are introduced in the proposed model. Assuming that a unit pulse excitation is imparted on the bottom end of drillstring, the pulse excitation responses obtained by the receivers $S_{1}$ and $S_{2}$ are, respectively, defined as $h_{1}(t)$ and $h_{2}(t)$. Similarly, assuming that a unit pulse excitation is imparted on the top end of drillstring, the pulse excitation responses collected by both receivers are, respectively, defined as $h_{1}^{\prime}(t)$ and $h_{2}^{\prime}(t)$. Accordingly, in terms of the channel model in Figure 2, the resulting signals $y_{1}$ and $y_{2}$ at the receivers $S_{1}$ and $S_{2}$ are

$$
\begin{aligned}
& y_{1}(t)=x(t) * h_{1}(t)+n_{s}(t) * h_{1}^{\prime}(t), \\
& y_{2}(t)=x(t) * h_{2}(t)+n_{s}(t) * h_{2}^{\prime}(t) .
\end{aligned}
$$

By fast Fourier transform (FFT) algorithm, (1) is written as

$$
\begin{aligned}
& Y_{1}(f)=H_{X_{1}}(f) X(f)+H_{N_{1}}(f) N_{s}(f), \\
& Y_{2}(f)=H_{X_{2}}(f) X(f)+H_{N_{2}}(f) N_{s}(f),
\end{aligned}
$$


TABLE 1: Dimensions of drillstring and single acoustic isolator.

\begin{tabular}{lccc}
\hline Structure & Component & Cross-sectional area $\left(\mathrm{m}^{2}\right)$ & Length $(\mathrm{m})$ \\
\hline \multirow{2}{*}{ Drillstring } & Drill pipe & $3.39 \times 10^{-3}$ & 9.11 \\
& Tool joint & $16.2 \times 10^{-3}$ & 0.475 \\
\hline \multirow{2}{*}{ Acoustic isolator } & Groove & The same as pipe body & $L_{g}$ (determined by simulation) \\
& Convex portion & The same as tool joint & $L_{c}$ (determined by simulation) \\
\hline
\end{tabular}

where $H_{X_{1}}(f), H_{X_{2}}(f), H_{N_{1}}(f)$, and $H_{N_{2}}(f)$ are the Fourier transforms of the functions $h_{1}(t), h_{2}(t), h_{1}^{\prime}(t)$, and $h_{2}^{\prime}(t)$ in the frequency domain, respectively.

Since the downward moving noise $n_{s}(t)$ disturbing acoustic signal extraction is a mixed signal of the noise and the data due to multiple reflections, the following result can be achieved by (2) to minimize the amount of noise being transmitted upward towards the surface:

$$
X(f)=\frac{H_{N_{2}}(f) Y_{1}(f)-H_{N_{1}}(f) Y_{2}(f)}{H_{X_{1}}(f) H_{N_{2}}(f)-H_{X_{2}}(f) H_{N_{1}}(f)} .
$$

It should be mentioned that the functions $h_{1}(t)$ and $h_{2}(t)$ in (1) can be determined in model simulations by solving the transient responses at two acoustic receivers $S_{1}$ and $S_{2}$ according to the one-dimensional extensional wave propagation equation in a periodic drillstring, when a unit impulse excitation is applied to the bottom end of drillstring. Likewise, the functions $h_{1}^{\prime}(t)$ and $h_{2}^{\prime}(t)$ can also be confirmed when a unit impulse excitation is applied to the top end of drillstring. In this way, $H_{X_{1}}(f), H_{X_{2}}(f), H_{N_{1}}(f)$, and $H_{N_{2}}(f)$ may be obtained by the Fourier transforms. In addition, the corresponding uplink and downlink channel responses in communication channel between surface receiver and downhole transmitter in real applications are sampled after a known chirp signal is generated at the downhole transmitter and the surface location of drillstring, respectively. The channel transfer function may be determined by crosscorrelating the received signal with the reference chirp signal, using a frequency spectrum of the received signal and a frequency spectrum of the reference chirp signal. Based on the determined transfer function, the functions $H_{X_{1}}(f)$, $H_{X_{2}}(f), H_{N_{1}}(f)$, and $H_{N_{2}}(f)$ in (3) may be eventually solved.

With inverse Fourier transform, the original excitation signal $x(t)$ can be computed by

$$
x(t)=\mathscr{F}^{-1}\left(\frac{H_{N_{2}}(f) Y_{1}(f)-H_{N_{1}}(f) Y_{2}(f)}{H_{X_{1}}(f) H_{N_{2}}(f)-H_{X_{2}}(f) H_{N_{1}}(f)}\right) .
$$

Then, the recovered signal $x(t)$ detected by dual receivers will be processed with a band-pass filter so that the frequency content is limited to the passband or bands which are used for data transmission. Also, it can eliminate the high-frequency content introduced by the algorithm computation.

2.2. Acoustic Isolator for Suppressing Uplink Downhole Noise. As mentioned above, downhole noise is also of concern in acoustic telemetry. A traditional response to this problem would be to place the acoustic telemetry device above

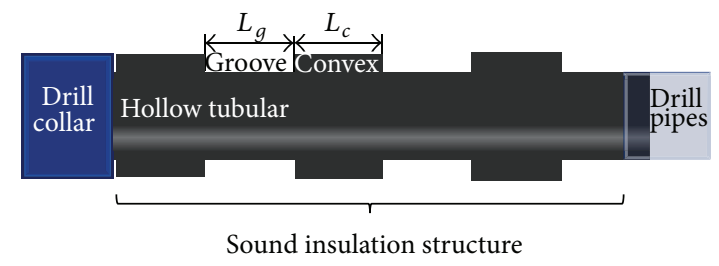

FIGURE 3: Structure diagram of a single acoustic isolator.

the BHA and simply direct the acoustic energy up the drillstring, away from the BHA components. Unfortunately, typical acoustic transmitters emit waves of equal magnitude in both "up" and "down" directions, and the downward travelling waves in particular may be reflected resulting in destructive interference with the upward travelling waves. In view of the banded spectral response characteristics of periodic drillstring, a single AIS with periodic annular grooves for acoustic insulation is designed analogously as demonstrated in Figure 3 on basis of the principle of mechanical vibration isolation.

It can be inserted between the transmitter and drill collar to impede unwanted strong reflections to the transmitter and subsequent to the receiver. From Figure 3, acoustic insulation performance of acoustic isolator is related to the length $L_{g}$ of groove, the length $L_{c}$ of the convex portion between adjacent grooves, and the number $N$ of grooves. According to the acoustic insulation theory in acoustic logging, in this paper both the lengths $L_{g}$ and $L_{c}$ are set equal to onequarter wavelength of the carrier frequency so that the center frequency to achieve the optimal noise attenuation can be determined. $L_{g}$ and $L_{c}$ are equal for simplicity of implementation and represented by the same letter $L$ in this paper, and the inner and outer diameters of both grooves and convex portions, respectively, have the same size as those of both pipes and tool joints as listed in Table 1. Same material for the AIS as drill pipes can be used.

Then, acoustic insulation performance can be represented by the transmission loss $\alpha$ as shown in the following equation:

$$
\alpha=20 \log _{10} \frac{A_{I}(f)}{A_{T}(f)}=20 \log _{10}\left(\frac{1}{T}\right),
$$

where $A_{I}(f)$ and $A_{T}(f)$ are, respectively, the incident and transmitted sound amplitude before and after the acoustic isolator in frequency domain, and the transmission coefficient $T$ relating to the transmission characteristics of acoustic isolator can be solved by using a Markov chain [18]. Assuming the reflection and transmission coefficients at tool joint $n$ 


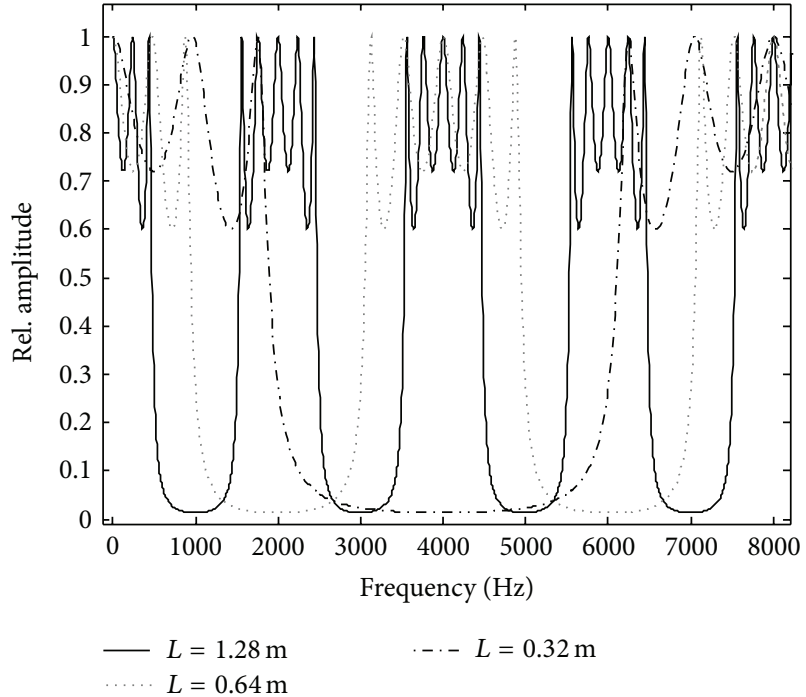

(a)

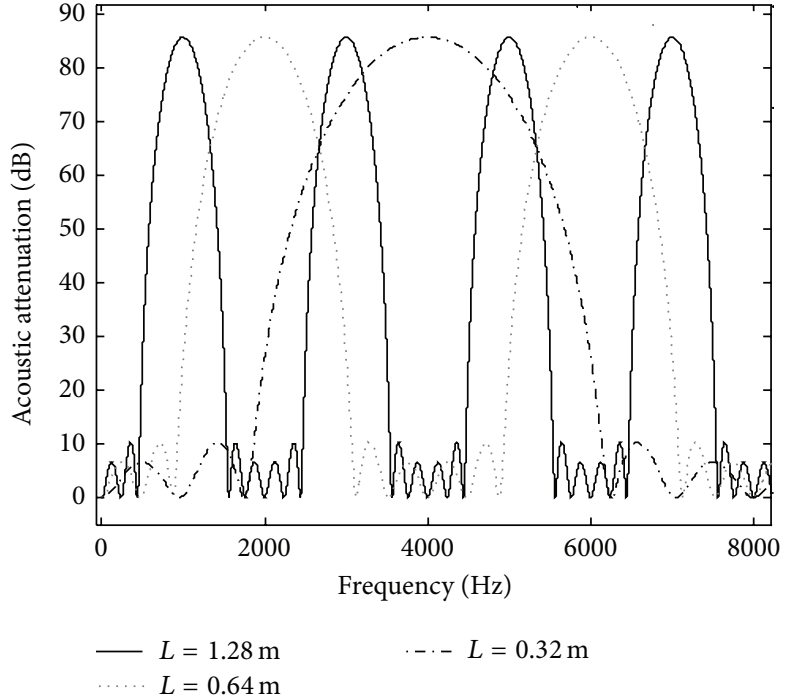

(b)

FIGURE 4: Simulation results of unit impulse frequency response and sound transmission loss for different single acoustic isolators. (a) Impulse frequency response and (b) sound transmission loss.

are denoted by $R_{n}$ and $T_{n}$, the transfer matrix $M_{n}$ of sound reflection and transmission at the tool joint between $n-1$ and $n$ is given by

$$
M_{n}=\left(\begin{array}{cc}
\frac{\left(T_{n}^{2}-R_{n}^{2}\right)}{T_{n}} & \frac{R_{n}}{T_{n}} \\
-\frac{R_{n}}{T_{n}} & \frac{1}{T_{n}}
\end{array}\right), \quad n=1,2, \ldots, N .
$$

Then, the transmission coefficient $T$ can be determined by setting transmission channel and boundary conditions. Referring to the basic structure described in Figure 3, a single AIS composed of three uniformly spaced grooves is used in numerical simulation where a unit pulse impact is imposed on the acoustic isolator. Then the impulse response and transmission loss of attenuated acoustic signal, passing through the acoustic isolator, can be resolved in frequency domain in accordance with (5) and (6) based on a Markov chain method. Figure 4 shows the impulse frequency responses and the sound transmission losses for different acoustic isolators when the lengths $L$ of groove and convex portions both are $1.28 \mathrm{~m}, 0.64 \mathrm{~m}$, and $0.32 \mathrm{~m}$, respectively. The result reveals that the acoustic isolator with uniformed-spaced periodic annular grooves also presents a banded and dispersive frequency response which has multiple passbands and stopbands. A complex peak pattern is produced in the frequency spectrum of each passband. And all the passbands have the same number of peaks. This number is related to the number of grooves in the acoustic isolator. As shown in Figure 4(a), the results obtained by an acoustic isolator with three grooves produce about three peaks exclusive of two peaks at the edges within each passband. The corresponding sound transmission losses of each AIS are displayed in Figure 4(b). In comparison, the signals in the stopbands have propagated

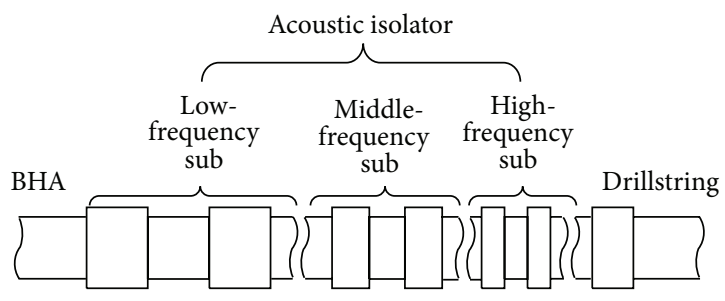

FIGURE 5: Structure diagram of a combined acoustic isolator.

with sharp reduction in amplitude, which explains why this sort of mechanical structural configuration can suppress uplink downhole noise. However, the center frequency at the first acoustic insulation stopband is approximately reduced from $4 \mathrm{kHz}$ to $1 \mathrm{kHz}$ when the lengths $L$ of groove and convex portions both increase to $1.28 \mathrm{~m}$ from $0.32 \mathrm{~m}$, resulting in a narrower acoustic insulation bandwidth in a maximum sound insulation of $80 \mathrm{~dB}$. In other words, the physical dimension of acoustic isolator can be adjusted to suit the noise reduction needed in a predetermined frequency range.

Unfortunately, the addition of further BHA components that cause more reflections often occurs during drilling operations. The performance of a single acoustic isolator is greatly limited in the amplitude and stopband bandwidth because of confined structural dimension. Thus, a combined acoustic isolator is beneficial. An example is shown in Figure 5. The combined acoustic isolator is made up of relatively low-, mid-, and high-frequency single subisolators in series connection. Among these three subisolators, the low-frequency sub is connected to the drill collar, and high-frequency sub is connected to the transmission assembly comprising an acoustic wave transmitter. Each sub has the following 


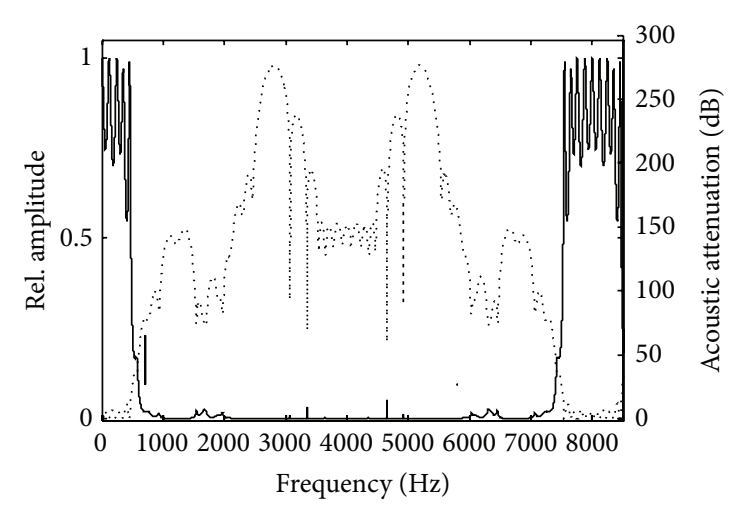

..... Acoustic attenuation characteristics - Frequency response

FIGURE 6: Simulation results of impulse frequency response and sound transmission loss for combined acoustic isolator.

characteristics: the passband of one sub is overlapped with the stopband of adjacent sub to have a wider stopband bandwidth than a single acoustic isolator. By referring back to Figure 4(b), the center frequency at the first acoustic insulation stopband is $1 \mathrm{kHz}, 2 \mathrm{kHz}$, and $4 \mathrm{kHz}$ when the length $L$ is set to $1.28 \mathrm{~m}, 0.64 \mathrm{~m}$, and $0.32 \mathrm{~m}$, respectively. Their sound insulation curves with a comb-like spectrum are overlapped with each other. For this reason, the frequencies $1 \mathrm{kHz}, 2 \mathrm{kHz}$, and $4 \mathrm{kHz}$ in the frequency range below $5 \mathrm{kHz}$ available for drillstring acoustic telemetry are used as the center frequencies of sound insulation at low-, mid-, and high-frequency bands, respectively. The impulse frequency responses and the sound transmission losses of the combined acoustic isolator are illustrated in Figure 6.

The impulse frequency response curve in Figure 6 also exhibits a distinctive feature characterized by the presence of passbands and stopbands. However, this feature is different from that of single acoustic isolator because the first stopband is widened and extended to about $0.5-7.5 \mathrm{kHz}$. The dotted sound insulation curve shows a similar bellshaped symmetrical distribution along the center frequency of $4 \mathrm{kHz}$ with a regular pattern of fine spikes. In comparison with the single acoustic isolator in Figure 4, the combined acoustic isolator has improved performance as a whole with an increased maximum sound insulation of $280 \mathrm{~dB}$. Therefore, by incorporating one or more devices capable of absorbing low-frequency acoustic energy into a drillstring, the amount of downhole drill bit noise entering the drillstring channel will be reduced. However, the maximum sound insulation occurs in the first stopband at the adjacent $5 \mathrm{kHz}$ instead of the center frequency of $4 \mathrm{kHz}$. This phenomenon is attributable to a standing-wave effect produced by the intersection of passbands and stopbands between adjacent subisolators. The imperfection in the structure will have an effect on noise suppression, so that a strong correlation exists between the structure-borne sound insulation and noise reduction distribution within the acoustic isolator.

\section{Transmission Performance Simulation}

Section 2 presents a detailed analysis of the joint acoustic echo and noise reduction method in drillstring channel. Barnes and Kirkwood in 1972 have indicated that the periodic structure formed by the pipe and tool joint sections results in frequency filtering and multiple echoes [8]. The acoustic impedance mismatches produced by discontinuous structure of drillstring also manifest surface and downhole noises through numerous spikes within each of the passbands of the transmission spectrum. The results of this analysis illustrate the importance of eliminating echo noises and enhancing the upward travelling acoustic telemetry signal collected by receiver. In this section, acoustic transmission performance along a finite-length drillstring is simulated by the timedomain finite-difference method using the following equation:

$$
\frac{\partial^{2} u}{\partial t^{2}}=\frac{E}{\rho} \frac{\partial^{2} u}{\partial x^{2}}
$$

where $E, \rho, u$, and $A$ are Young's modulus, density, longitudinal displacement, and cross-sectional area of drillstring, respectively.

Equation (7) is in the form of a classical wave equation. Attenuation mechanisms which are present in the actual field environment are not included in the analysis. These mechanisms include viscous dissipation of energy into the surrounding drill mud and physical contact with the formation and well casing. However, since the periodic structure will cause a redistribution and not merely a reduction of amplitude and spreading of the wave, the effects of the propagating echo and noise in drillstring are focused primarily on in this paper.

The time-domain algorithms of linear, one-dimensional wave equations have the advantages for many problems with complex combinations of geometry and boundary conditions. The drillstring is divided into segments. Within each segment it is assumed that the density and the cross-sectional area are constant. The position of mesh point $x_{n}$ is labeled with an integer $n$. The position within the segment between meshes points $x_{n}$ and $x_{n+1}$ is labeled with a number $n+$ $1 / 2$. The displacement field $u(x, t)$ at the mesh point $x_{n}$ is approximated by a discrete set of values $u_{n}^{j}$. To improve the stability of the algorithm, uniform critical time step $\Delta t$ is used. By using simple-centered differences, (7) is rewritten as

$$
u_{n}^{j+1}+u_{n}^{j-1}=\frac{2\left(z_{n+1 / 2} u_{n+1}^{j}+z_{n-1 / 2} u_{n-1}^{j}\right)}{z_{n+1 / 2}+z_{n-1 / 2}},
$$

where $j$ denotes the time $j \Delta t ; \Delta t$ is critical time step; $n$ denotes the position $x_{n}$, and $z$ is the string impedance for axial (or extensional) waves. By specifying the boundary conditions

$$
\begin{aligned}
& u_{n}^{j} \quad(j=0,1 ; n=0,1,2, \ldots, N), \\
& u_{n}^{j} \quad(j=0,1,2, \ldots, N ; n=0, N),
\end{aligned}
$$




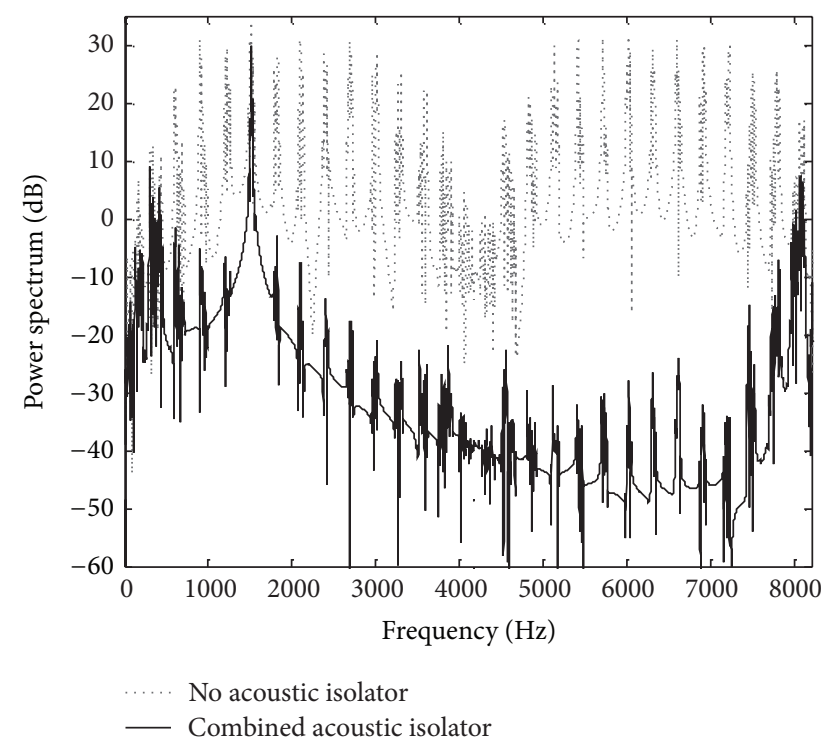

Figure 7: Simulation results of sinusoidal impulse frequency response in the absence and presence of combined acoustic isolator.

acoustic transmission transient behaviors along the drillstring can be analyzed.

3.1. Acoustic Insulation Performance. Due to the periodic structure of drillstring, ten drill pipes separated by nine tool joints as listed in Table 1 are used in the transient simulation. The whole length of the drillstring is about $95.4 \mathrm{~m}$. Referring back to Figure 1, the acoustic transmitter is located near the bottom end of drillstring. A combined acoustic isolator with a length of $13.4 \mathrm{~m}$ is placed below the acoustic transmitter. The top end of the drillstring is modeled as a fixed end because of the mass of the blocks suspending the drillstring or the mass of the drilling platform. According to the obtained capacity curve of the uplink channel versus the SNR by using the measured bit and surface noise data and mathematical model for an approximately $2 \mathrm{~km}$ drillstring in reference [19], an uplink capacity may be reached for an SNR slightly smaller than $0 \mathrm{~dB}$ considering practically achievable data rates of several hundred bits/s. In this case, assuming the upwardgoing noise from the bit to be additive and Gaussian, the Gaussian white noise $n_{d}(t)$ with zero mean and a variation of 8 is acted on the lower end of the acoustic isolator. From the patterns of passbands and stopbands in the simulated drillstring channel, a continuous sine wave pulse of $1.52 \mathrm{kHz}$ frequency with unit amplitude in the passband region is applied at the transmitter. Thus an SNR of $-1.17 \mathrm{~dB}$ at downhole transmitter in simulation is yielded to be in close approximation to the actual condition. Two acoustic receivers $S_{1}$ and $S_{2}$ spaced one-quarter wavelength apart are placed on the first pipe at the top of the drillstring to record two signals. The two-path signals are processed to retrieve the raw excitation signal filtered by echo canceller according to (4). Then the retrieved signal response in frequency domain is shown in Figure 7, where the combined acoustic isolator is either used or not used. The results show that the received signal has an appearance of white noise, which implies that the upward-going noise interferes with the transmitted data. In the absence of combined acoustic isolator, the channel noise as a whole is nearly as intense as the response of signal intensity at the exciting frequency of $1.52 \mathrm{kHz}$ in the 0 to $8 \mathrm{kHz}$ frequency range. Since the transmitted signal is covered by the noise, the absence of the acoustic isolator may result in no signal at all being received at surface. However, an increasingly better signal-to-noise ratio can be obtained if the combined acoustic isolator is available. The solid line in Figure 7 indicates that the signal intensity at $1.52 \mathrm{kHz}$ excitation frequency is higher than the noise intensity by more than $50 \mathrm{~dB}$. Also, the combined acoustic isolator makes the noise level efficiently reduced by $30-40 \mathrm{~dB}$ in the average noise level. Therefore, an appropriate acoustic isolator, formed along the drillstring below a downhole acoustic transmitter, is effective in reflecting "up" propagating waves originating from below the isolator, hence further protecting the acoustic wave source from possible deleterious interference.

3.2. Modulated Signal Transmission. It has been discovered that certain discrete frequency passbands permit the transmission of an acoustic signal with a minimum level of attenuation in the assembled drillstring [20]. It is essential that acoustic signal transmission performance is evaluated by sending the modulated data along the drillstring within the optimum passband. The binary data is generally sent in three basic ways, including amplitude-shift keying (ASK) modulation, frequency-shift keying (FSK) modulation, and phase-shift keying (PSK) modulation [21]. And currently simple communication modulation schemes, such as ASK, FSK, PSK, and their derivatives, are still commonly applied to the actual downhole telemetry systems. Therefore, from the view of the principle experiment and the performance test, we derive a ASK, FSK, or PSK modulation to be applied to a base band digital signal, respectively. A packet of 50 bit modulated pseudorandom binary code sequence is brought to bear on the left end of drillstring. The carrier frequency and data rate are, respectively, configured to be $1.52 \mathrm{kHz}$ and $450 \mathrm{bit} / \mathrm{s}$. Each of the digital modulation schemes is tested by simulation for ten times to evaluate the performance of acoustic data communication. The simulation conditions, concerning the drillstring, combined acoustic isolator, upward-going noise $n_{d}(t)$ and dual-receiver configurations in Section 3.1, are shared in this section. Moreover, the additive white Gaussian noise with zero mean and a variation of 8 is acted on the top end of drillstring, to simulate the effects of downward-going surface noise $n_{s}(t)$. In this case, the mixed signal and noise coexist in the periodic fading simulation channel.

First, in order to prove the availability of AEC, the influence factors related to acoustic isolator and the noise $n_{d}(t)$ can be ignored. The output of receiver $S_{1}$ at the optimal position is recommended as single-receiver signal. Then the BER curves along a 95.4 meter drillstring for the three different types of modulation in either a single- or dualreceiver mode are presented in Figure 8. The simulation results in Figure 8(a) show that there is no obvious difference 


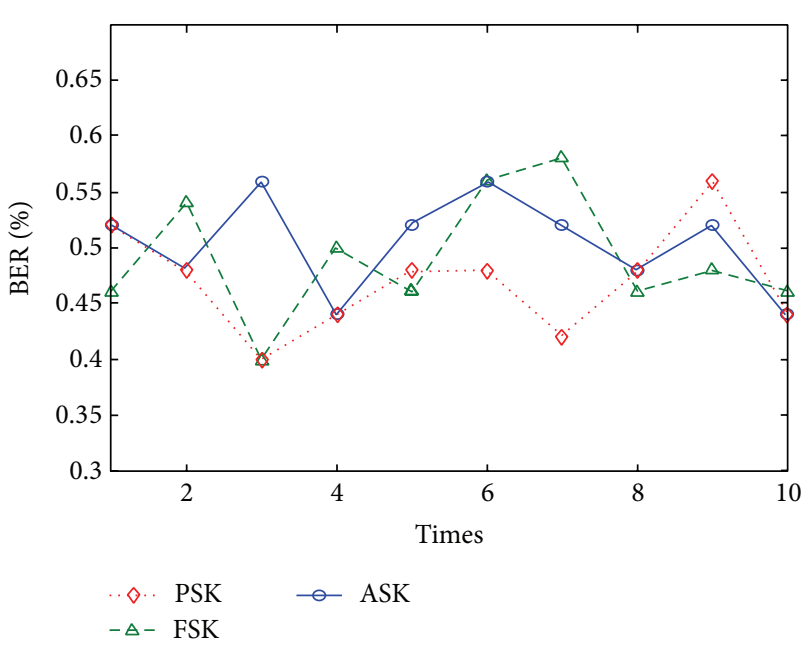

(a)

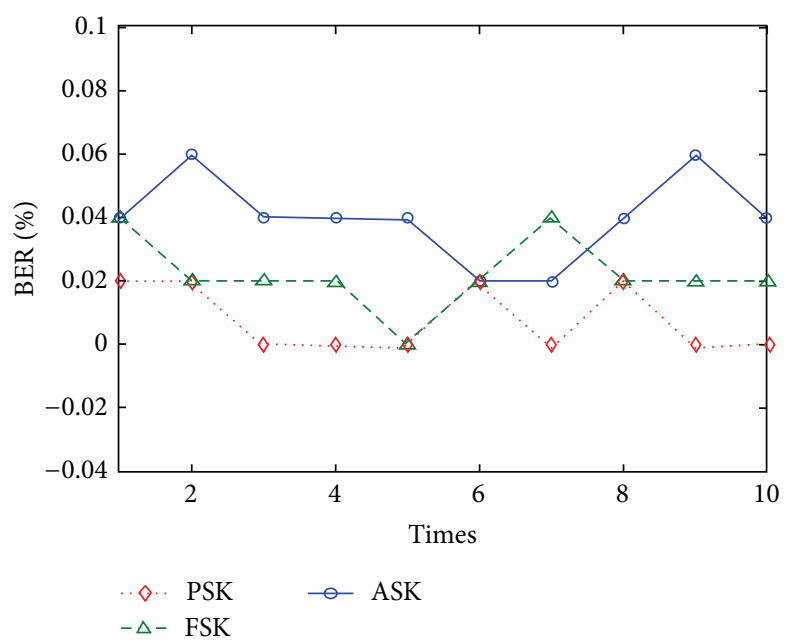

(b)

FIgURE 8: The BER simulation curves of ASK-, FSK-, and PSK-modulated data in either a single- or a dual-receiver mode. (a) Single-receiver mode and (b) dual-receiver mode.

TABLE 2: The average BER for three basic modes of modulation.

\begin{tabular}{lccc}
\hline Boundary condition & ASK & FSK & PSK \\
\hline $\begin{array}{l}\text { Without acoustic } \\
\text { isolator and noise } n_{d}(t)\end{array}$ & $4 \%$ & $2.2 \%$ & $0.8 \%$ \\
\hline $\begin{array}{l}\text { Without acoustic } \\
\text { isolator but considering } \\
\text { the noise } n_{d}(t)\end{array}$ & $17.6 \%$ & $17.2 \%$ & $4 \%$ \\
\hline $\begin{array}{l}\text { Considering acoustic } \\
\text { isolator and the noise } \\
n_{d}(t)\end{array}$ & $6.4 \%$ & $7 \%$ & $0.6 \%$ \\
\hline
\end{tabular}

between the BERs in single-receiver mode. They vary within the range of $47 \%-50.4 \%$. Nevertheless, the average BER is significantly reduced by more than $94 \%$ when the dualreceiver mode is employed in Figure 8(b). In general, PSK is less susceptible to errors than the other two modulation schemes, while it requires more complex phase recovery process. Especially, the average BER of PSK-modulated pulse signal is reduced by up to $0.8 \%$ from $47 \%$ if the dual-receiver scheme is substituted for single-receiver scheme. This proves that the appropriate use of two acoustic receivers with proper modulation scheme can offer a higher SNR and data rate in multipath channel of drillstring.

Subsequently, the combined acoustic isolator and the noise $n_{d}(t)$ are introduced to consider the functions of AEC and AIS together in order to assess the performance of sound insulation and echo suppression. The BER curves along the drillstring in the absence and the presence of combine acoustic isolator are presented in Figure 9. In comparison with the BER curves in Figure 8(b), the average BER for the above three kinds of modulation under different boundary conditions is set forth in Table 2 .

As mentioned earlier, the echo noise tends to reduce the SNR, regardless of the noise source. The acoustic transmission rate and telemetry range are limited because of the poor

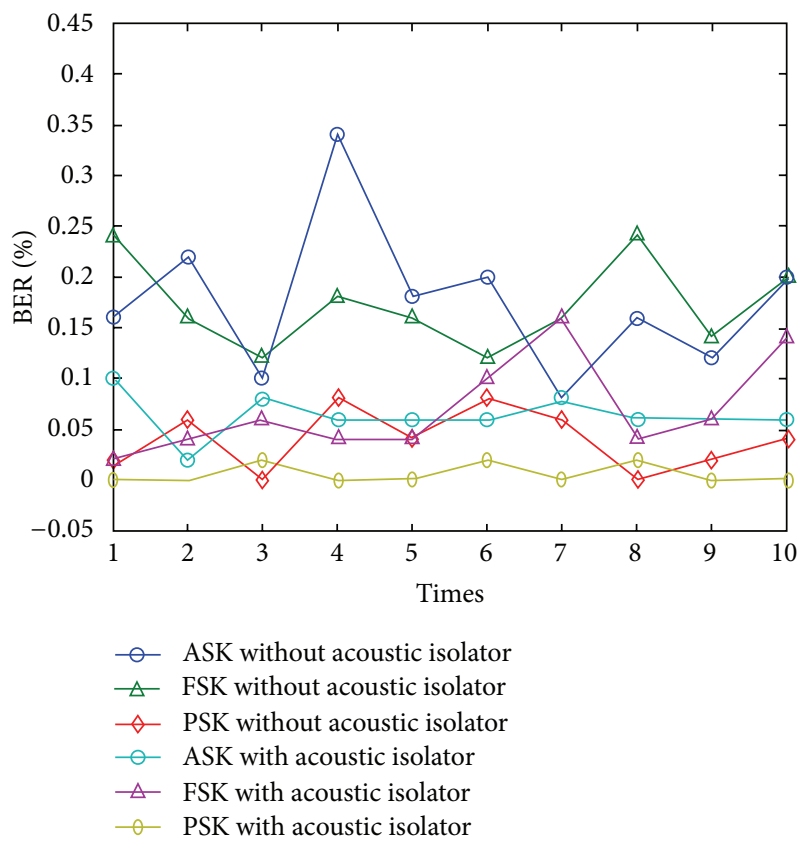

FIGURE 9: The BER simulation curves of ASK-, FSK-, and PSKmodulated data in the absence and the presence of combine acoustic isolator.

BER. It can be inferred from Table 2 that it is necessary for downhole acoustic telemetry to insert a proper acoustic isolator below the transmitter because the drill bit noise and the strong BHA reverberations deteriorate the retrieval of transmitted signal. For example, the average BER becomes much worse in the absence of acoustic isolator when the noise $n_{d}(t)$ is transmitted through the drillstring and then arrives at the receiver. The BER for ASK or FSK modulation is increased by more than $17 \%$, but PSK modulation with 
a relative lower BER of $4 \%$ is more superior to others. After the proposed combined acoustic isolator is used, the worse situation is restrained with a sharply improved BER of $6.4 \%$, $7 \%$, and $0.6 \%$ for the aforementioned three basic modulation modes. In particular, PSK modulation represents a much better BER performance which slightly surpasses the BER of $0.8 \%$ in the case ignoring acoustic isolator and the noise $n_{d}(t)$ as shown in Figure 8(b). This phenomenon may result not only from the selected phase sensitive demodulation threshold, but also from the signal enhancement caused by acoustic isolator. It can be concluded that PSK modulation is more adapted for the stable channel conditions because of the more sensitivity to phase noise, while FSK modulation is an attractive modulation when the phase changes too quickly to be tracked. In contrast, ASK modulation suffers from the highest BER. However, when properly implemented, ASK can actually also exhibit a lower probability of error versus SNR for two receivers, especially limited bandwidth channel. In conclusion, the BER curves in Figure 9 reveal that acoustic transmission performance is dramatically improved by the proposed new scheme of joint dual-wave receiving and sound insulation. It can allow the ringing to fall to tolerable levels, along with a proper combined intersymbol interference (ISI) and modulation coding scheme, which further contributes to the cancellation of acoustic interferences in drillstring waveguide.

\section{Conclusions}

Acoustic telemetry is a promising technique to transmit the downhole information to the surface in a drilling operation. However, the situation is made even more challenging by the significant surface and downhole noise generally experienced. The periodic structure of drillstring as transmission medium also makes the problems of echo noise and signal distortion more severe. With regard to the transmission path of acoustic echo and noise along the drillstring, a joint acoustic echo and noise suppression scheme using an acoustic echo canceller and an acoustic insulation structure is provided for attenuating the unwanted multiple disturbances before they can interfere with the required upward travelling waves at the receiver. Although three types of waves, including extensional, bending, and torsional waves, will propagate in a drillstring in realistic drilling conditions, the lateral motion induced by bending waves easily couples with surrounding drilling fluids and extensional waves, resulting in large attenuation and strong downhole noise. Unfortunately, torsional waves undergo stronger reflections at tool joints, since their reflection depends on the ratio of the polar moments of inertia of cross-sectional areas and most of drilling noises reside in the torsional mode. Hence, extensional waves provide superior transmission performance for communication. By using a finite-differential algorithm for one-dimensional propagation of extensional waves, acoustic transmission performances along the periodic pipe structure channel are simulated either in a single receiver or in dual receivers and either by having an acoustic isolator or not above the downhole transmitter. The results verify that the appropriate use of proposed scheme can offer a lower bit error ratio and an improved transmission performance in multipath channel of drillstring by removing those undesired waves in the "up" and "down" directions.

\section{Conflict of Interests}

The authors declare that there is no conflict of interests regarding the publication of this paper.

\section{Acknowledgments}

The authors thank the National Natural Science Foundation of China (nos. 50905095, 61121003) and Changjiang Scholars and Innovative Research Team in University for supporting this research.

\section{References}

[1] L. Duncan, "MWD ranging-a hit and a miss," Oil Gas European Magazine, vol. 39, no. 1, pp. 24-26, 2013.

[2] T. Ding and L. Cheng, "Coreless electromagnetic couplingbased drillstem telemetry using dual electronic gauges," SPE Production and Operations, vol. 22, no. 1, pp. 128-134, 2007.

[3] L. Cheng, T. Ding, and W. Peng, "An experimental rig for nearbit force measurement and drillstring acoustic transmission of BHA," Measurement: Journal of the International Measurement Confederation, vol. 44, no. 4, pp. 642-652, 2011.

[4] Y. Shinmoto, E. Miyazaki, K. Wada, and M. Yamao, "Development of a continuous directional coring system for deep-sea drilling," SPE Drilling and Completion, vol. 27, no. 1, pp. 139-144, 2012.

[5] L. Gao, D. Finley, W. Gardner et al., "Acoustic telemetry can deliver more real-time downhole data in underbalanced drilling operations," in Proceedings of the IADC/SPE Drilling Conference, pp. 485-490, February 2006.

[6] Q. M. Li, B. Clark, S. B. Mehta et al., "Bi-directional drill string telemetry system for measurement and drilling contro," Patent EP20060813296, 2006.

[7] D. S. Drumheller, "Coupled extensional and bending motion in elastic waveguides," Wave Motion, vol. 17, no. 4, pp. 319-327, 1993.

[8] T. G. Barnes and B. R. Kirkwood, "Passbands in acoustic transmission in idealized drill string," Journal of the Acoustical Society of America, vol. 51, no. 5, pp. 1606-1608, 1972.

[9] M. E. Reeves, P. L. Camwell, and J. McRory, "High speed acoustic telemetry network enables real-time along string measurements, greatly reducing drilling risk," in Proceedings of the SPE-Offshore Europe Oil and Gas Conference and Exhibition, vol. 1, pp. 458-469, September 2011.

[10] J. W. Rector III and B. P. Marion, "The use of drill-bit energy as a downhole seismic source," Geophysics, vol. 56, no. 5, pp. 628$634,1991$.

[11] D. S. Drumheller and D. D. Scott, "Circuit for echo and noise suppression of acoustic signals transmitted through a drill string," Patent US 5274606, 1993.

[12] F. Poletto, "Use of dual waves for the elimination of reverberations in drill strings," Journal of the Acoustical Society of America, vol. 111, no. 1 I, pp. 37-40, 2002. 
[13] S. Sinanović, D. H. Johnson, and W. R. Gardner, "Directional propagation cancellation for acoustic communication along the drill string," in Proceedings of the IEEE International Conference on Acoustics, Speech and Signal Processing, vol. 4, pp. 521-524, May 2006.

[14] R. Meehan, "Method and apparatus for suppressing drillstring vibrations," Patent US 6535458, 2003.

[15] D. S. Drumheller, P. L. Camwell, A. R. Dopf, and D. W. Logan, "Acoustic isolator," Patent US 8270251, 2012.

[16] V. V. Shah, E. J. Linyaev, D. G. Kyle, and W. R. Gardner, "Low frequency acoustic attenuator for use in downhole applications," Patent US 7210555, 2007.

[17] A. Ghasemloonia, D. G. Rideout, and S. D. Butt, "Vibration analysis of a drillstring in vibration-assisted rotary drilling: finite element modeling with analytical validation," Journal of Energy Resource Technology, vol. 135, no. 3, pp. 032902-103290218, 2013.

[18] N. J. C. Lous, S. W. Rienstra, and I. J. B. F. Adan, "Sound transmission through a periodic cascade with application to drill pipes," Journal of the Acoustical Society of America, vol. 103, no. 5, pp. 2302-2311, 1998.

[19] S. Sinanovic, Limits of acoustic waveguide communication [Ph.D. thesis], Rice University, 2006.

[20] D. S. Drumheller, "Attenuation of sound waves in drill strings," Journal of the Acoustical Society of America, vol. 94, no. 4, pp. 2387-2396, 1993.

[21] J. D. Oetting, "A comparison of modulated techniques for digital radio," IEEE transactions on communications systems, vol. 27, no. 12, pp. 1752-1762, 1979. 

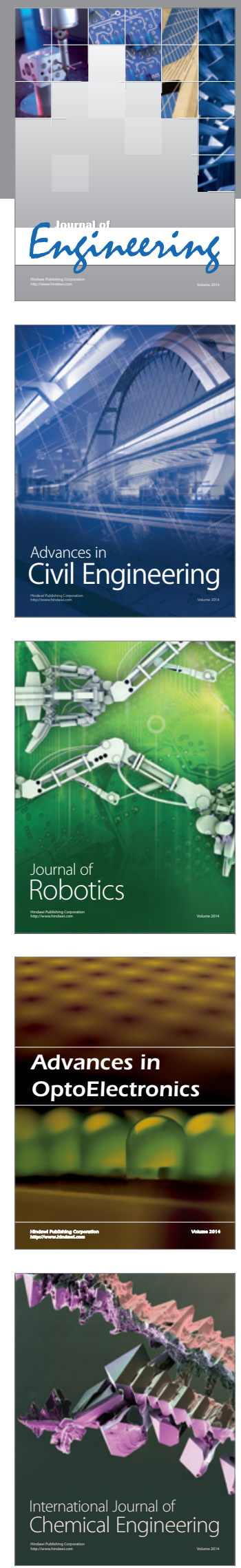

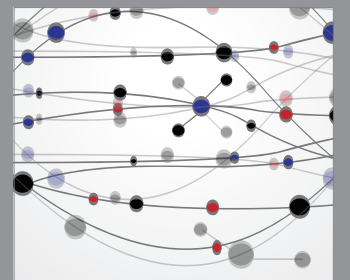

The Scientific World Journal
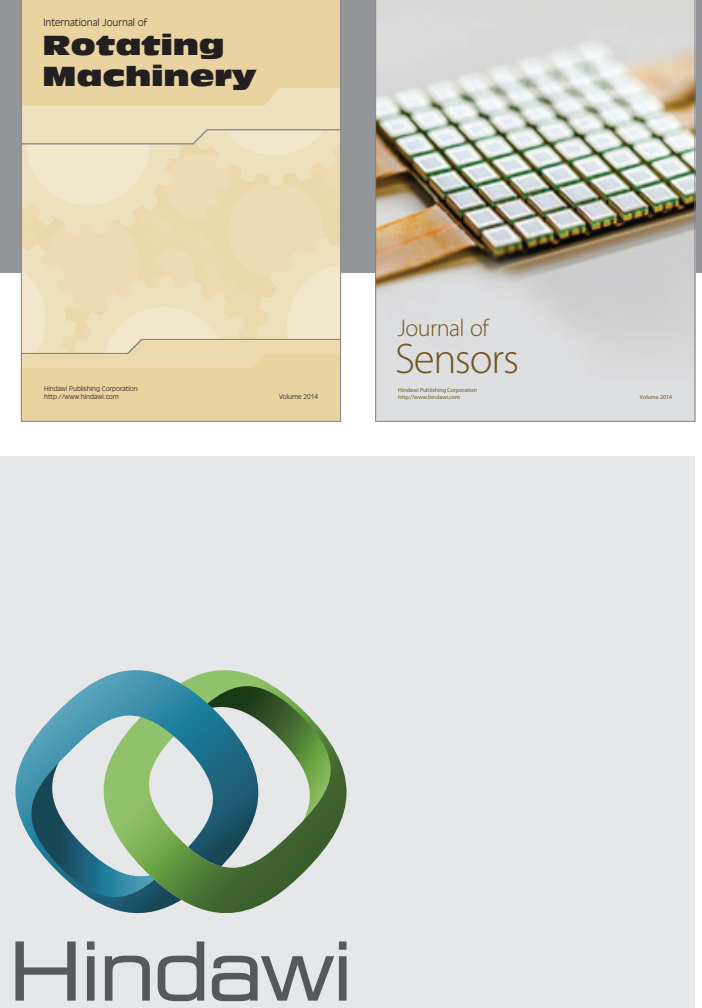

Submit your manuscripts at http://www.hindawi.com
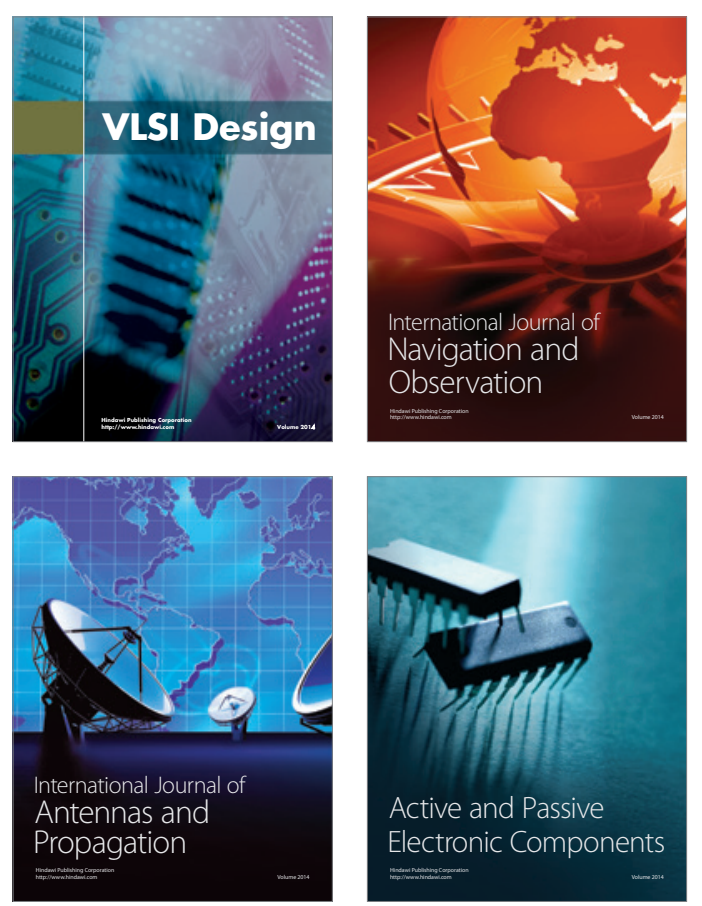
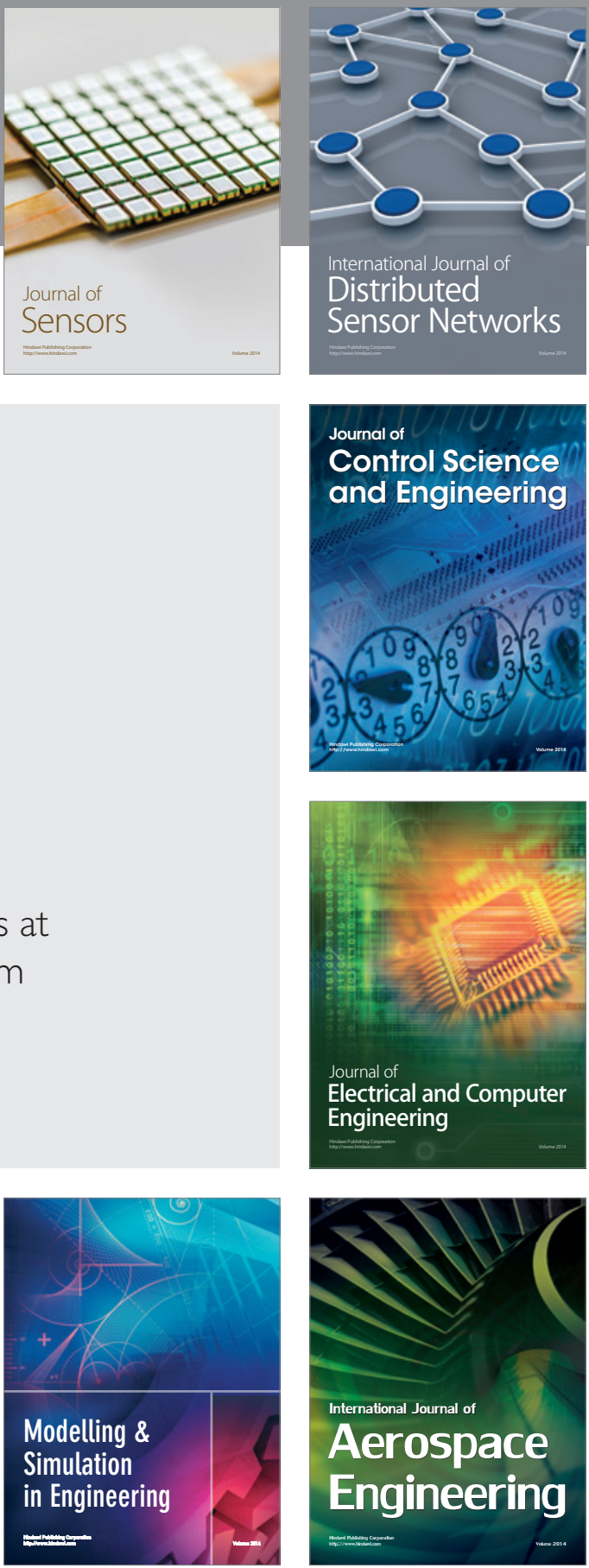

Journal of

Control Science

and Engineering
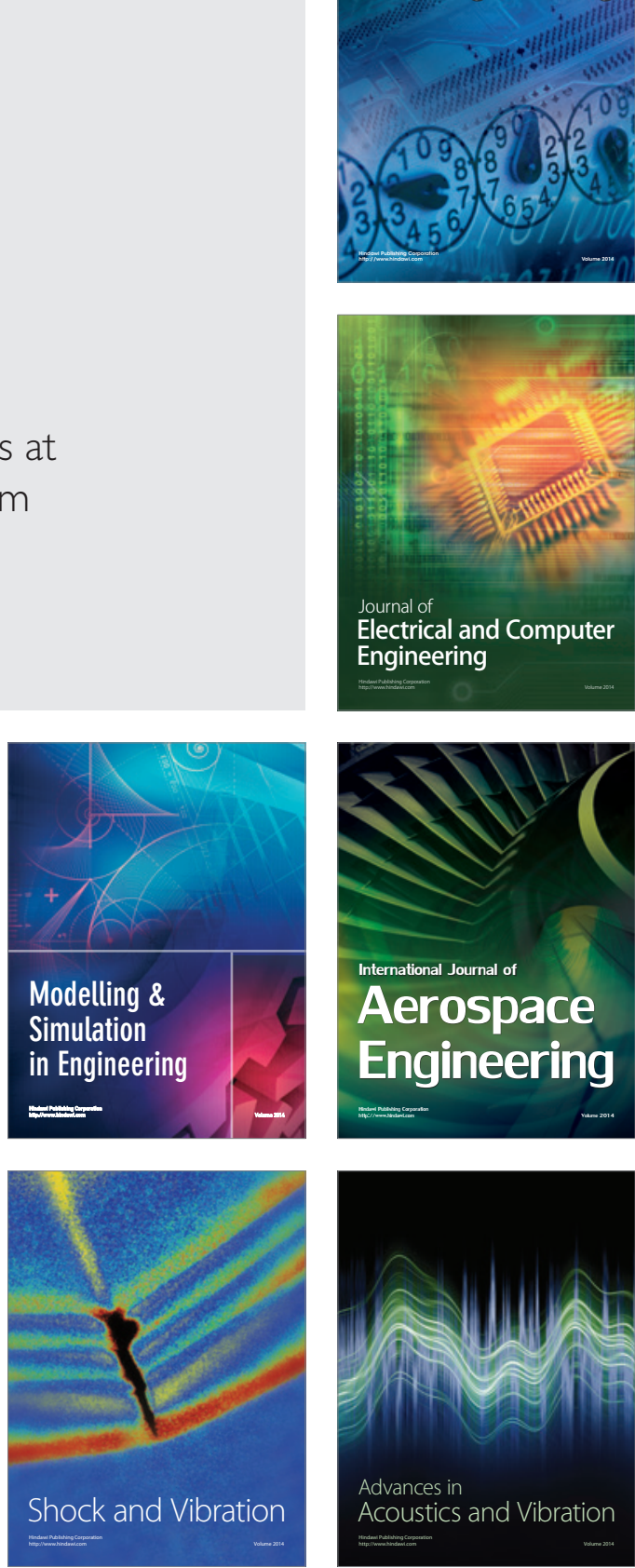